INPLASY

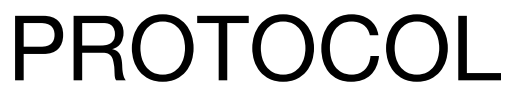

To cite: Si et al. Pregnancy for patients with autoimmune hepatitis: a systematic review and meta-analysis. Inplasy protocol 202060036. doi: 10.37766/inplasy2020.6.0036

Received: 10 June 2020

Published: 10 June 2020

Corresponding author: Tengfei Si

tengfei.si@kcl.ac.uk

Author Affiliation:

Institute of Liver Studies,King's

College Hospital

Support: This study is supported by State Scholarship Fund (201808310051): China Scholarship Council; The Henry Lester Trust; Surgical Funds, King's College Hospital Charity; Visiting scholar fund, Shanghai University of Traditional Chinese Medicine

Review Stage at time of this submission: Data analysis.

Conflicts of interest:

The authors declare that they have no competing interests.

\section{Pregnancy for patients with autoimmune hepatitis: a systematic review and meta-analysis}

Si, T1; Graham, J2; Zhao, D³; Huang, Z4; Ma, Y5; Heneghan, M6.

Review question / Objective: Will pregnancy increase the risk of relapse in patients with autoimmune hepatitis? And what effect does pregnancy have on AlH women and newborns? Condition being studied: To date, research surrounding the exact effect of pregnancy on AIH patients' liver function remains limited. Pregnancy is marked by significant temporal changes of a variety of hormones during all three trimesters which consequently, regulate and promote tolerance of maternal immune system. We therefore want to know for AlH women of childbearing age, whether pregnancy is safe for both mother and infants. And to further understand how it will affect patients' liver function and how to guide the medicine more safely during pregnancy.

INPLASY registration number: This protocol was registered with the International Platform of Registered Systematic Review and Meta-Analysis Protocols (INPLASY) on 10 June 2020 and was last updated on 10 June 2020 (registration number INPLASY202060036).

\section{INTRODUCTION}

Review question / Objective: Will pregnancy increase the risk of relapse in patients with autoimmune hepatitis? And what effect does pregnancy have on AlH women and newborns?

Condition being studied: To date, research surrounding the exact effect of pregnancy 
on AlH patients' liver function remains limited. Pregnancy is marked by significant temporal changes of a variety of hormones during all three trimesters which consequently, regulate and promote tolerance of maternal immune system. We therefore want to know for AlH women of childbearing age, whether pregnancy is safe for both mother and infants. And to further understand how it will affect patients' liver function and how to guide the medicine more safely during pregnancy.

\section{METHODS}

Search strategy: A systematic search was conducted according to the Cochrane Handbook. The primary search strategy was as follow: ((gravidity[Title/Abstract]) OR (gestation[Title/Abstract])) OR (pregnancy[Title/Abstract])) OR ( $p r e g n a n t[T i t$ e / A b s t act ]) AND( (autoimmune [Title/Abstract]) AND( ((hepatitis [Title/Abstract]) OR (liver disease [Title/Abstract]) ) OR (AIH [Title/ Abstract]) OR (AILD [Title/Abstract])). The searching cut-off date was 12 December 2019. We also checked the reference lists of all identified studies for additional eligible data.

Participant or population: Pregnant women with autoimmune hepatitis.

Intervention: Natural pregnancy or in- vitro fertilization.

\section{Comparator: None.}

Study designs to be included: All published studies including abstracts from conferences, primary research, clinical trials (randomised controlled trials, nonrandomised trials).

Eligibility criteria: All studies mentioned above reporting the pregnancy in AlH women. The language of published literatures is limited to English.

Information sources: Databases including PubMed, Embase, Cochrane library and Science Citation Index Expanded were searched to collect all available studies about pregnancy in AlH women. We also checked the reference lists of all identified studies for additional eligible data.

Main outcome(s): The primary outcomes include the relapse of $\mathrm{AlH}$, complication after delivery as well as the occurrence of preterm delivery.

Additional outcome(s): Comparison of clinical indicators of patients before and after delivery including liver function indicators (AST, ALT, GGT, etc.), immune function indicators (IgG, IgM, etc.) and so on.

Data management: Data extraction from each study was performed by two authors independently. Patients' basic characteristics, changes in liver function during pregnancy, results of laboratory tests after delivery, postoperative complications, and outcomes of new-borns were exacted from each study using a predesigned data extraction form. For the missing information, we tried to contact the authors of original articles. During the process of data extraction, any disagreement was resolved by discussion or with a third reviewer if necessary.

Quality assessment / Risk of bias analysis: The study quality assessment /risk of bias analysis was conducted by two reviewers independently. The Newcastle-Ottawa Scale (NOS) was performed to evaluate the quality of observational study, while the Risk of bias graph and summary suggested by Cochrane Handbook were used for randomly controlled studies. During the process of quality assessment, any disagreement was resolved by discussion or with a third reviewer if necessary.

Strategy of data synthesis: Review Manager (version 5.3, The Cochrane Collaboration, Copenhagen) recommended by Cochrane Collaboration was used to perform the meta-analysis. Dichotomous variables were tested by Risk Ratio (RR) with a $95 \%$ confidence interval $(\mathrm{Cl})$, and continuous variables were tested by the standardized mean difference (SMD) with a $95 \% \mathrm{Cl}$. Random effect model was used for 
all calculations. Heterogeneity between studies was tested by chi-squared test (with significance set at $p>0.1$ ) and Isquared test. Funnel plots were also used to investigate the publication bias if sufficient studies existed. A value of $p<0.05$ was considered statistically significant.

Subgroup analysis: Subgroup analysis was performed to identify the source of heterogeneity when appropriate.

Sensibility analysis: None.

Language: English.

Country(ies) involved: Authors were from China and United Kingdoms.

Other relevant information: Clinical data of pregnant AlH patients from King's College Hospital was also collected.

Keywords: Pregnancy, autoimmune hepatitis, meta-analysis.

Contributions of each author:

Author 1 - Tengfei Si - Study Design, Data collection and analysis, Result discussion, Manuscript writing, Proof reading and editing.

Author 2 - Jonathon Graham - Data collection and analysis, Result discussion, Proof reading and editing.

Author 3 - Dong Zhao - Data collection and analysis, Result discussion, Manuscript writing, Proof reading and editing.

Author 4 - Zhenlin Huang - Data collection and analysis, Result discussion, Manuscript writing, Proof reading and editing.

Author 5 - Yun Ma - Result discussion, Manuscript writing, Proof reading and editing.

Author 6 - Michael Heneghan - Study Design, Result discussion, Proof reading and editing. 